\title{
Graphical Representation of Time Use Diaries in Stata: ATUS Tempograms
}

\author{
Kamila Kolpashnikova \\ University of Oxford \\ kamila.kolpashnikova@sociology.ox.ac.uk
}

Acknowledgements: This project has received funding from

the European Union's Horizon 2020 research and innovation programme

under the Marie Sklodowska-Curie grant agreement No 892101

(awardee: Kamila Kolpashnikova).

ver. 08.2020

\section{Introduction}

Although Stata is not the best package to use for time use graphical representation, such as for tempograms ${ }^{1}$, it is nonetheless possible. In this paper, I will demonstrate how to create tempograms using the original American Time Use Survey data from the US Bureau of Labor Statistics ${ }^{2}$. For this project, the 2003-2018 sample of diaries is used (file names: atusact0318 and atussum0318).

Additionally, I identify the bottleneck, where the performance of Stata's underlying functions could be optimised to improve the work with time-use data for researchers who use Stata.

\section{Downloading and Preparing Data}

The data used for this visualisations are publicly available from the Bureau of Labor Statistics website. The 2003-2018 American Time Use Survey files can be found at the following link: https://www.bls.gov/tus/data files-0318.htm. I use two files from ATUS:

1. atusact0318 (https://www.bls.gov/tus/special.requests/atusact-0318.zip) - this file contains diary day activity information. For details on the activity coding and data collection, please visit the BLS website.

2. atussum0318 (https://www.bls.gov/tus/special.requests/atussum-0318.zip) - this file contains information about the summary of activity durations. However, for this project, I used it to extract information on weekday and year. The original name for the weekday of the diary day variable is tudiaryday and for the year variable - tuyear.

BLS provides original dictionary files within the compressed .zip files linked above. Before using the code in this project, please transform data into .dta files using BLS codes in the provided links and save both as .dta extension files.

The biggest bottleneck for the present project was reshape command. There are custom solutions to this problem, which can be found online, but it might not solve the problem for the use of the native Stata reshape

\footnotetext{
${ }^{1}$ at of the time of this project, $\mathrm{R}$ has a faster performance both in wrangling and plotting (TraMineR) of the time use sequences on bigger datasets such as ATUS 2003-2018.

${ }^{2}$ https://www.bls.gov/tus/
} 
command.

\section{Step 1.}

- open atusact0318.dta file

- use a new variable called ID for tucsaseid

- sort by ID and the diary activity number

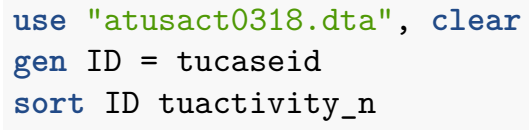

\section{Step 2.}

- create a variable (in minutes) when the activity starts (start) and finishes (stop) using duration variable and the sequence number of the activity

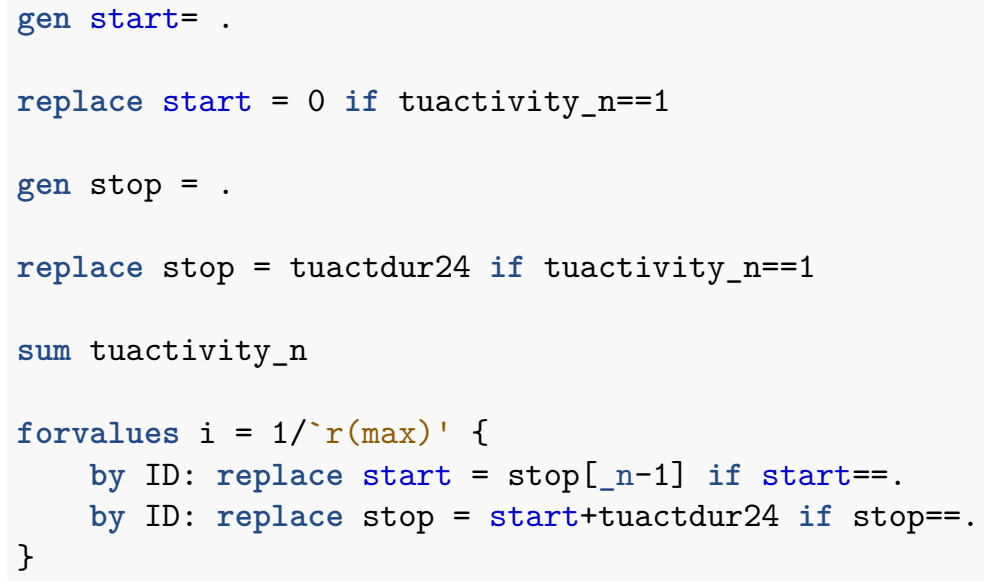

\section{Step 3.}

- combine activity codes into larger categories:

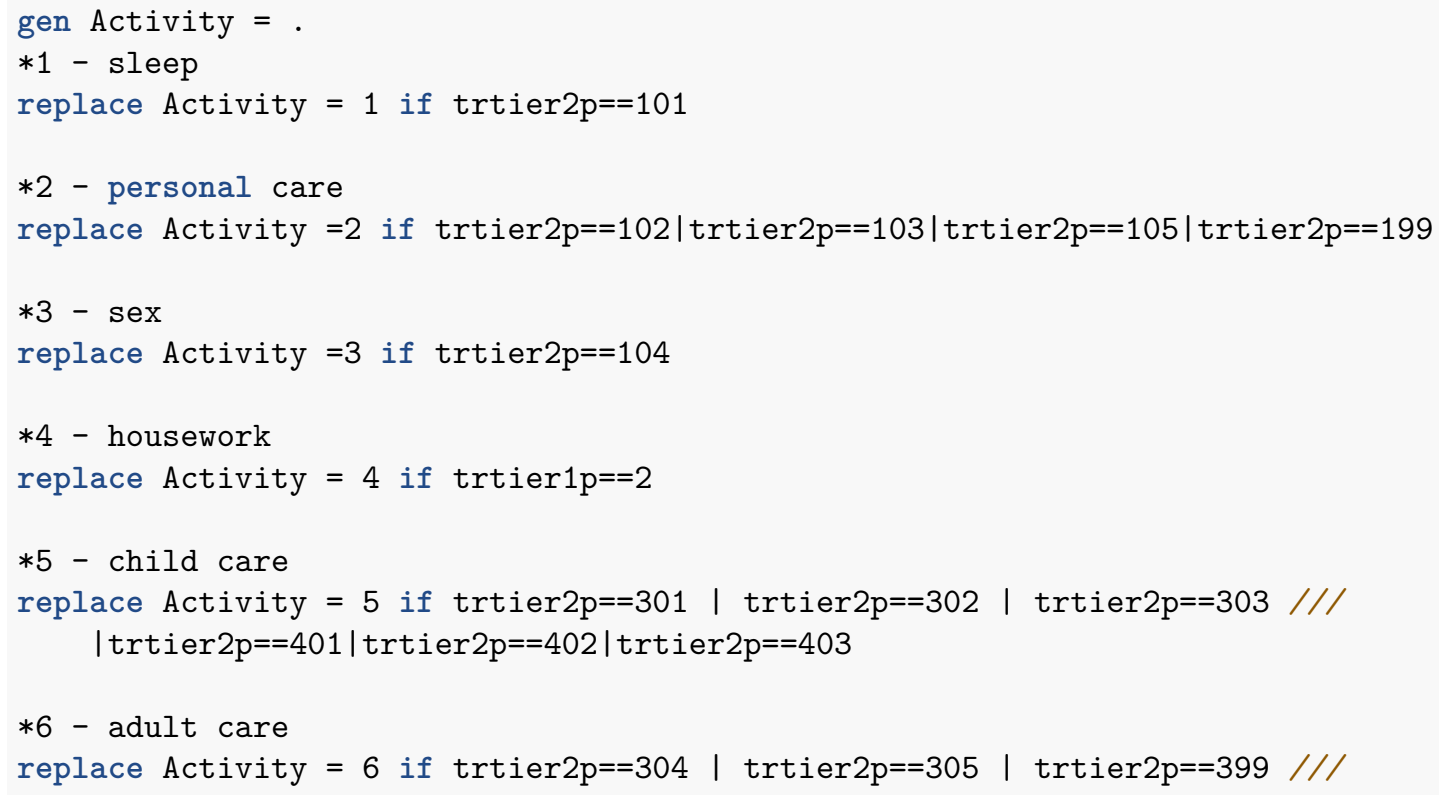


$|\operatorname{trtier} 2 \mathrm{p}==404| \operatorname{trtier} 2 \mathrm{p}==405 \mid \operatorname{trtier} 2 \mathrm{p}==499$

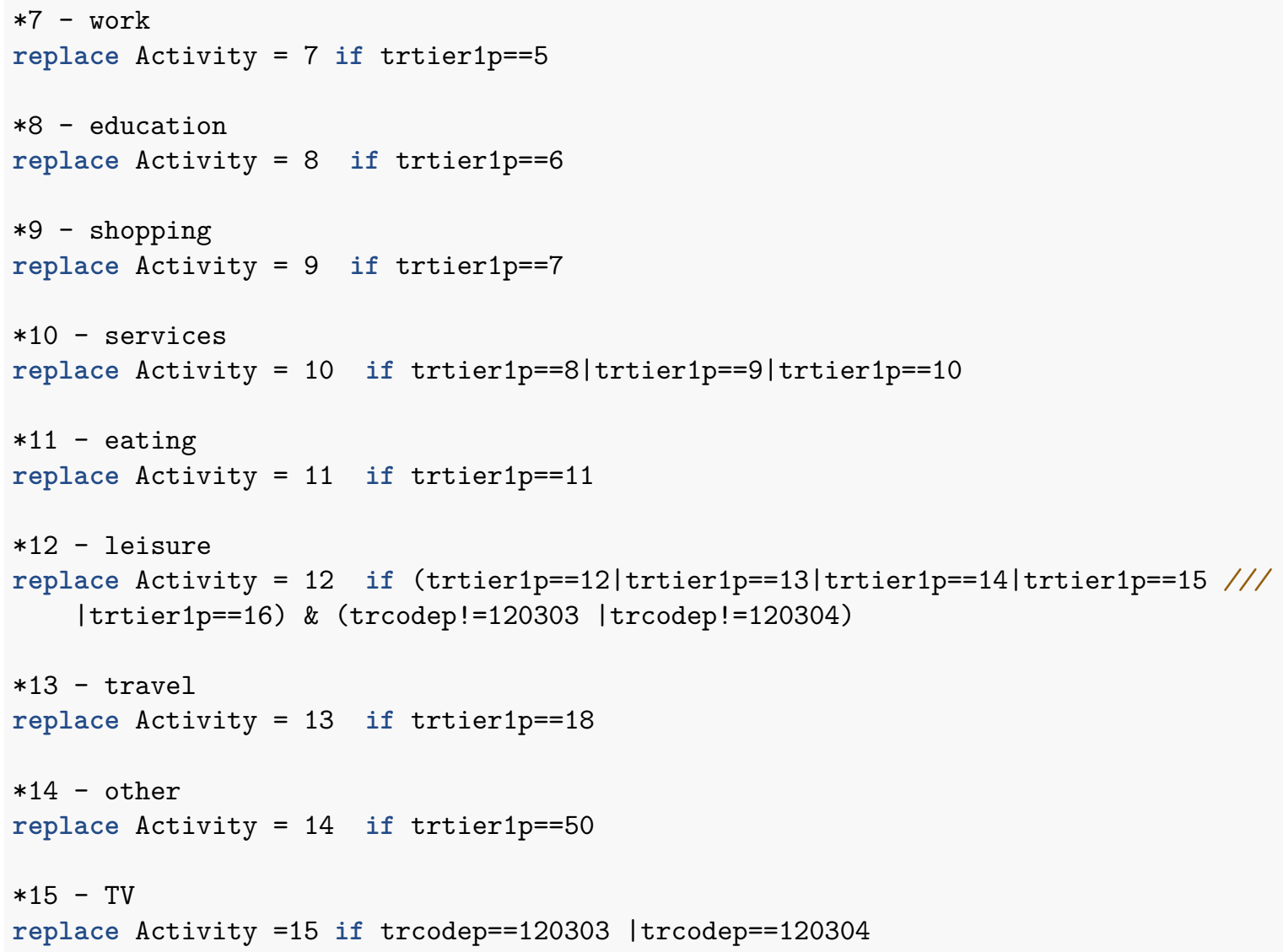

\section{Step 4.}

- keep only the variables that will be needed for the next steps

keep tucaseid ID tuactivity_n Activity start tuactdur24

\section{Step 5.}

- create the number of observations 1440 for each ID by expanding the duration variable. The resulting number of observations should be $289,657,440$

expand tuactdur 24

\section{Step 6.}

- create a minutes step identifier (time slot) by ID

sort ID tuactivity_n start

by ID: gen time $={ }_{-} \mathrm{n}$

\section{Step 7.}

- delete non-essential variables. Keep only ID, time, and activity variables 


\section{Step 8.}

- reshape wide and save

WARNING: This step will take a very long time. It took on Windows laptop (Intel Core i5, 16GB RAM) - 15 hours, and on MacBook Pro (8-Core Intel Core i9, 64GB DDR4) - 6 hours. This is the narrowest bottleneck in the code. There are custom solutions to this problem on online forums, but the solution specific to time use data is needed. ${ }^{3}$

reshape wide Activity, i(ID) j(time)

save "ATUS 0318 sequences.dta", replace

Above will save a file in the following form:

\begin{tabular}{|c|c|c|c|c|c|c|c|c|c|c|c|c|c|c|}
\hline & ID & Activity 1 & Activity 2 & Activity3 & Activity4 & Activity5 & Activity 6 & Activity 7 & Activity8 & Activity9 & Activity10 & Activity11 & Activity 12 & Activi \\
\hline 1 & 20030100013280 & Leisure & Leisure & Leisure & Leisure & Leisure & Leisure & Leisure & Leisure & Leisure & Leisure & Leisure & Leisure & L \\
\hline 2 & 20030100013344 & Sleep & Sleep & Sleep & Sleep & Sleep & Sleep & Sleep & Sleep & Sleep & Sleep & sleep & Sleep & \\
\hline 3 & 20030100013352 & Sleep & Sleep & Sleep & Sleep & Sleep & Sleep & Sleep & Sleep & Sleep & Sleep & Sleep & Sleep & \\
\hline 4 & 20030100013848 & Sleep & Sleep & Sleep & Sleep & Sleep & Sleep & Sleep & Sleep & Sleep & Sleep & Sleep & Sleep & \\
\hline 5 & 20030100014165 & Sleep & sleep & Sleep & Sleep & Sleep & sleep & Sleep & sleep & Sleep & Sleep & Sleep & Sleep & \\
\hline 6 & 20030100014169 & Sleep & Sleep & Sleep & Sleep & Sleep & Sleep & Sleep & sleep & Sleep & Sleep & Sleep & Sleep & \\
\hline 7 & 20030100014209 & Sleep & Sleep & Sleep & Sleep & Sleep & Sleep & Sleep & Sleep & Sleep & Sleep & Sleep & Sleep & \\
\hline 8 & 20030100014427 & Sleep & Sleep & Sleep & Sleep & Sleep & Sleep & Sleep & sleep & Sleep & Sleep & Sleep & Sleep & \\
\hline 9 & 20030100014550 & Sleep & Sleep & Sleep & Sleep & Sleep & Sleep & Sleep & Sleep & Sleep & Sleep & Sleep & Sleep & \\
\hline 10 & 20030100014758 & sleep & sleep & Sleep & Sleep & Sleep & sleep & sleep & sleep & sleep & Sleep & Sleep & Sleep & \\
\hline 11 & 20030100014928 & sleep & sleep & Sleep & Sleep & Sleep & sleep & Sleep & Sleep & sleep & Sleep & Sleep & Sleep & \\
\hline 12 & 20030100015106 & Sleep & Sleep & Sleep & Sleep & Sleep & Sleep & Sleep & Sleep & Sleep & Sleep & Sleep & Sleep & \\
\hline 13 & 20030100015322 & Sleep & Sleep & Sleep & sleep & Sleep & Sleep & Sleep & Sleep & Sleep & Sleep & Sleep & Sleep & \\
\hline 14 & 20030100015390 & Sleep & sleep & Sleep & sleep & sleep & Sleep & Sleep & sleep & Sleep & Sleep & Sleep & Sleep & \\
\hline 15 & 20030100015490 & sleep & sleep & Sleep & sleep & sleep & sleep & sleep & sleep & Sleep & Sleep & Sleep & sleep & \\
\hline 16 & 20030100015890 & sleep & sleep & Sleep & sleep & sleep & sleep & Sleep & sleep & Sleep & Sleep & sleep & Sleep & \\
\hline 17 & 20030100015891 & Sleep & sleep & Sleep & sleep & sleep & sleep & Sleep & sleep & Sleep & Sleep & Sleep & Sleep & \\
\hline 18 & 20030100015941 & Sleep & Sleep & Sleep & sleep & Sleep & sleep & Sleep & Sleep & sleep & Sleep & Sleep & Sleep & \\
\hline 19 & 20030100015986 & Sleep & Sleep & Sleep & Sleep & Sleep & Sleep & Sleep & sleep & Sleep & Sleep & Sleep & Sleep & \\
\hline 20 & 20030100016070 & sleep & sleep & sleep & sleep & sleep & sleep & sleep & sleep & sleep & Sleep & sleep & Sleep & \\
\hline 21 & 20030101030010 & sleep & Sleep & Sleep & sleep & Sleep & Sleep & Sleep & sleep & sleep & Sleep & Sleep & Sleep & \\
\hline 22 & 20030101030056 & Sleep & Sleep & Sleep & Sleep & Sleep & Sleep & Sleep & Sleep & Sleep & Sleep & Sleep & Sleep & \\
\hline 23 & 20030101030074 & Sleep & Sleep & Sleep & Sleep & Sleep & Sleep & Sleep & Sleep & Sleep & Sleep & Sleep & Sleep & \\
\hline 24 & 20030101030082 & Sleep & Sleep & Sleep & Sleep & Sleep & Sleep & Sleep & sleep & Sleep & Sleep & Sleep & Sleep & \\
\hline 25 & 20030101030093 & Sleep & Sleep & Sleep & Sleep & Sleep & Sleep & Sleep & Sleep & Sleep & Sleep & Sleep & Sleep & \\
\hline & 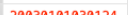 & chan & ch... & cha. & c1m... & c1... & c.... & cho. & cin. & c1... & clam & c1m. & c1... & \\
\hline
\end{tabular}

Figure 1: Sequences Table

\section{Labeling Activities and Adding Variables}

\section{Step 9.}

- define and add labels to activity variables

- save changes

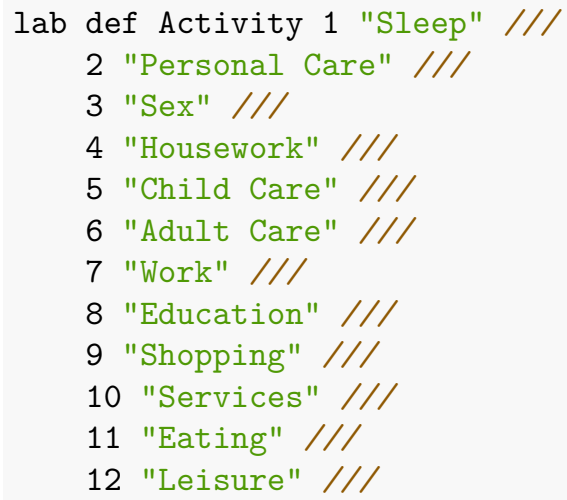

\footnotetext{
${ }^{3}$ I will probably develop it soon. Stay tuned.
} 


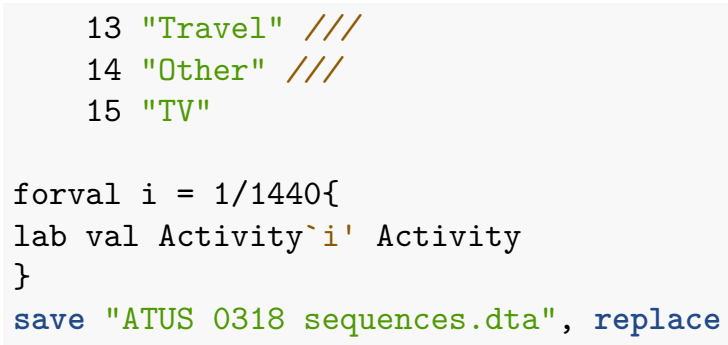

\section{Step 10.}

- extract year and weekday variables from atussum0318.dta file

- merge with the existing file

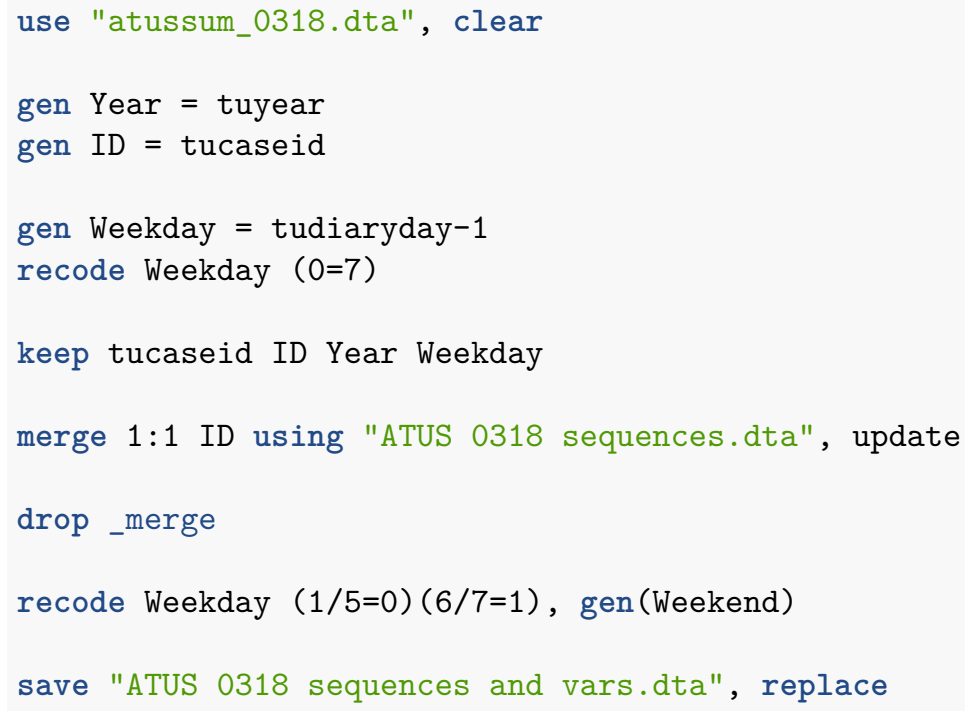

\section{Creating Tempogram Data Table}

\section{Step 11.}

The files for tempograms are based on diary sequences. They contain the time slot variable and the number of observations in each activity. The structure is presented in Figure 2.

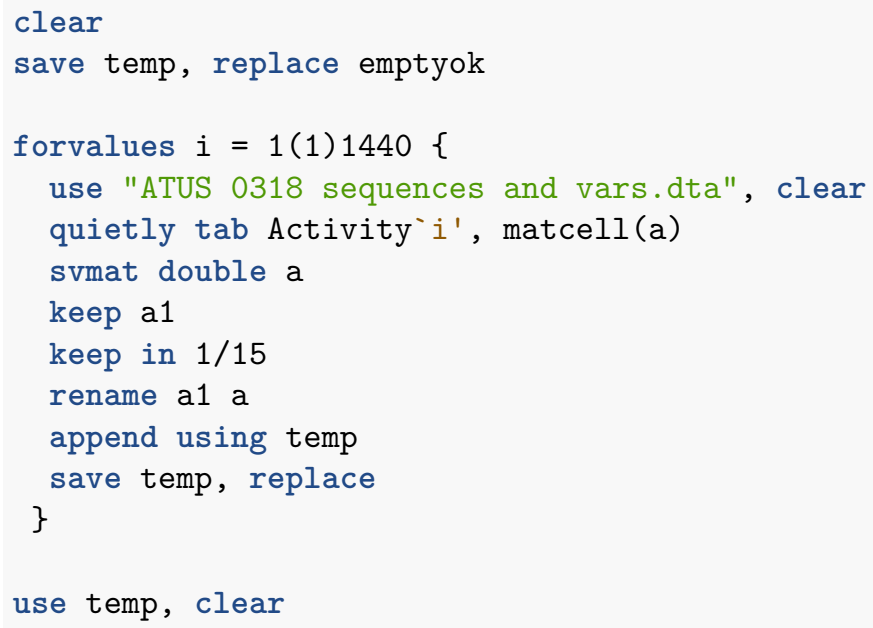


recode a $(.=0)$

\section{Step 12.}

- install seq package from ssc, if you don't have it installed. This package will help to transform one column variable (from the commands above, temp) into a sequence object.

ssc install seq

\section{Step 13}

- declare a sequence object and reshape wide

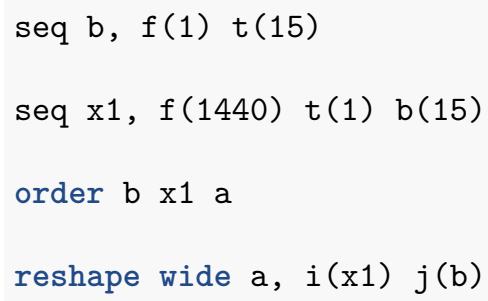

Figure 2 shows the resulting tempogram data table.

\begin{tabular}{|c|c|c|c|c|c|c|c|c|c|c|c|c|c|c|c|c|}
\hline & $\mathrm{x} 1$ & $\mathrm{a} 1$ & $\mathrm{a} 2$ & a3 & a4 & a5 & a6 & a7 & a8 & a9 & a10 & a11 & a12 & a13 & a14 & a15 \\
\hline 1 & 1 & 185695 & 3574 & 55 & 1697 & 915 & 145 & 3444 & 77 & 33 & 35 & 849 & 1868 & 919 & 183 & 1662 \\
\hline 2 & 2 & 185529 & 3631 & 54 & 1711 & 916 & 141 & ActiIs4ty & eş7a & $15_{35}$ & 35 & 877 & 1897 & 928 & 184 & 1686 \\
\hline 3 & 3 & 185549 & 3602 & 53 & 1677 & 916 & 140 & 3456 & 77 & 36 & 35 & 888 & 1910 & 926 & 184 & 1702 \\
\hline 4 & 4 & 185566 & 3596 & 50 & 1651 & 916 & 139 & 3456 & 77 & 37 & 33 & 893 & 1916 & 925 & 184 & 1712 \\
\hline 5 & 5 & 185575 & 3598 & 50 & 1638 & 916 & 139 & 3456 & 77 & 38 & 33 & 889 & 1923 & 923 & 183 & 1713 \\
\hline 6 & 6 & 185603 & 3596 & 47 & 1525 & 902 & 130 & 3463 & 79 & 38 & 34 & 910 & 1957 & 934 & 185 & 1748 \\
\hline 7 & 7 & 185604 & 3590 & 48 & 1522 & 901 & 130 & 3463 & 79 & 38 & 34 & 913 & 1961 & 932 & 185 & 1751 \\
\hline 8 & 8 & 185605 & 3583 & 48 & 1518 & 902 & 129 & 3465 & 79 & 39 & 34 & 911 & 1963 & 933 & 185 & 1757 \\
\hline 9 & - $\quad 9$ & 185605 & 3582 & 48 & 1515 & 903 & 128 & 3465 & 79 & 39 & 34 & 913 & 1961 & 933 & 185 & 1761 \\
\hline 10 & Times & $\mathrm{t}_{1856-6 \mathrm{~B}} \mathrm{C}$ & 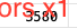 & 48 & 1516 & 901 & 128 & 3465 & 79 & 39 & 34 & 914 & 1960 & 932 & 185 & 1762 \\
\hline 11 & 11 & 185680 & 3527 & 48 & 1449 & 872 & 131 & 3483 & 79 & 37 & 35 & 896 & 1999 & 927 & 181 & 1807 \\
\hline 12 & 12 & 185686 & 3521 & 48 & 1441 & 870 & 130 & 3486 & 79 & 37 & 35 & 895 & 2006 & 926 & 181 & 1810 \\
\hline 13 & 13 & 185688 & 3522 & 48 & 1426 & 871 & 128 & 3488 & 79 & 35 & 35 & 899 & 2012 & 928 & 181 & 1811 \\
\hline 14 & 14 & 185690 & 3518 & 48 & 1424 & 870 & 127 & 3489 & 79 & 34 & 35 & 898 & 2015 & 928 & 181 & 1815 \\
\hline 15 & 15 & 185690 & 3519 & 48 & 1419 & 870 & 127 & 3489 & 79 & 34 & 35 & 901 & 2014 & 929 & 181 & 1816 \\
\hline 16 & 16 & 185657 & 3417 & 44 & 1437 & 809 & 133 & 3526 & 78 & 36 & 35 & 889 & 2057 & 983 & 183 & 1867 \\
\hline 17 & 17 & 185658 & 3407 & 42 & 1437 & 807 & 131 & 3531 & 78 & 35 & 35 & 893 & 2058 & 986 & 182 & 1871 \\
\hline 18 & 18 & 185668 & 3408 & 42 & 1426 & 804 & 129 & 3533 & 78 & 34 & 35 & 892 & 2059 & 987 & 183 & 1873 \\
\hline 19 & 19 & 185668 & 3405 & 42 & 1430 & 802 & 129 & 3533 & 78 & 34 & 35 & 887 & 2060 & 990 & 184 & 1874 \\
\hline 20 & 20 & 185668 & 3406 & 42 & 1427 & 803 & 129 & 3533 & 78 & 34 & 35 & 889 & 2060 & 989 & 184 & 1874 \\
\hline 21 & 21 & 185736 & 3203 & 42 & 1422 & 722 & 124 & 3582 & 78 & 42 & 34 & 901 & 2108 & 1049 & 194 & 1914 \\
\hline
\end{tabular}

Figure 2: Tempogram Table

\section{Step 14.}

- label the time slot variable

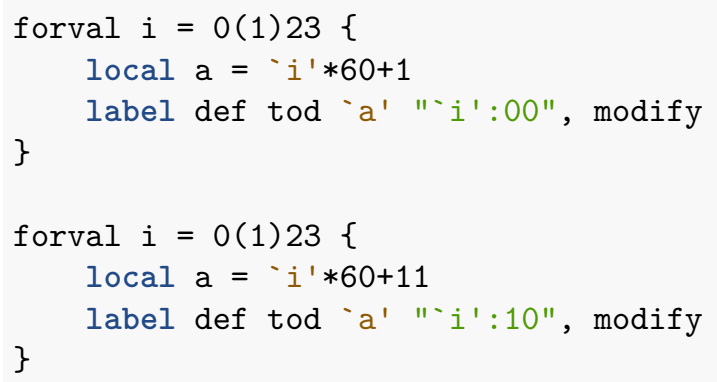




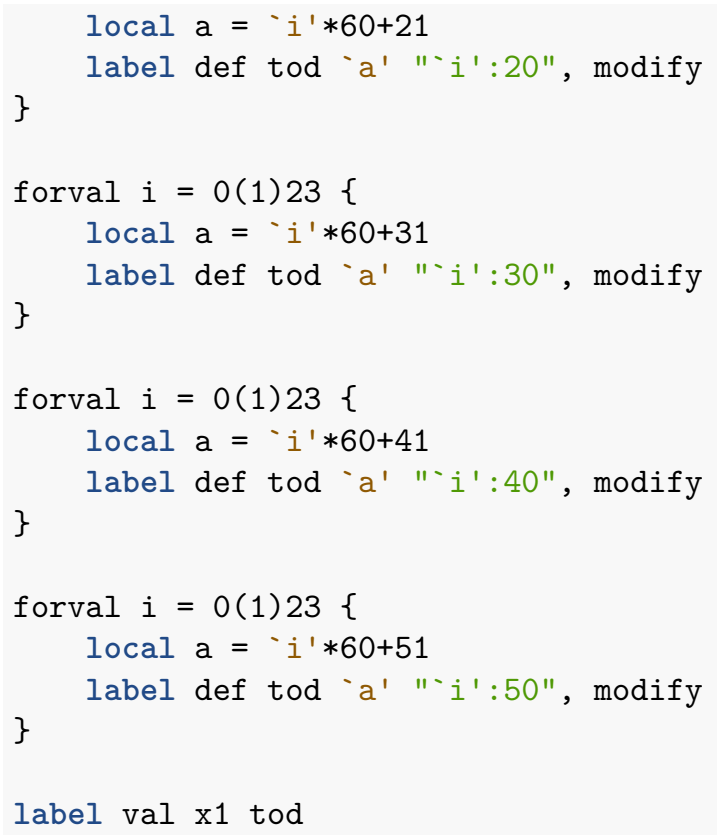

\section{Aggregate Activities}

\section{Step 15.}

We need to aggregate times for activities in the order that we want to present them because twoway area plots area graphs over each other. To make areas visible above each other, we need to aggregate them. Rules: - the activity that is meant to be on the bottom of the tempogram comes first and is not aggregated with others (in my examples, it is a1, sleep) - the activity that is meant to be on the top of the tempogram should come the last and combines all other activities (here, it's a14, other)

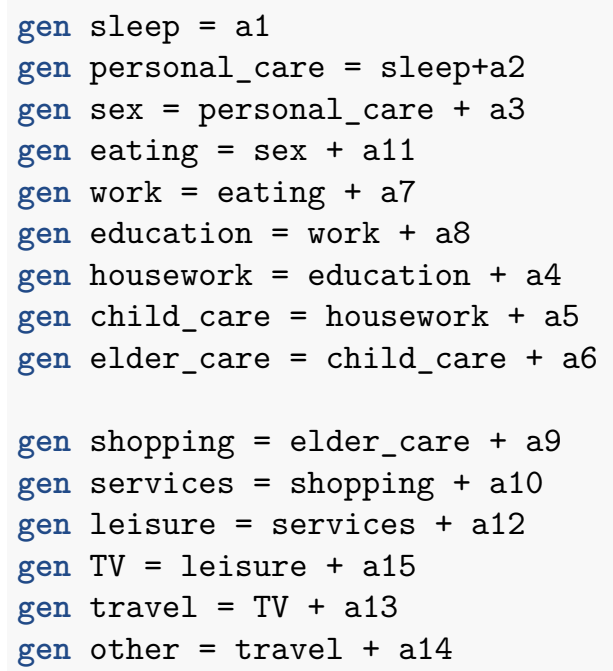

\section{Plotting Tempograms}

\section{Example 1. Plotting Tempogram of all activities in a day}

- use twoway area 
- order the entry of activity variables from the top ('other') to the bottom activity ('sleep'). x1 is the time slot activity.

- xlabel allows to add specific ticks

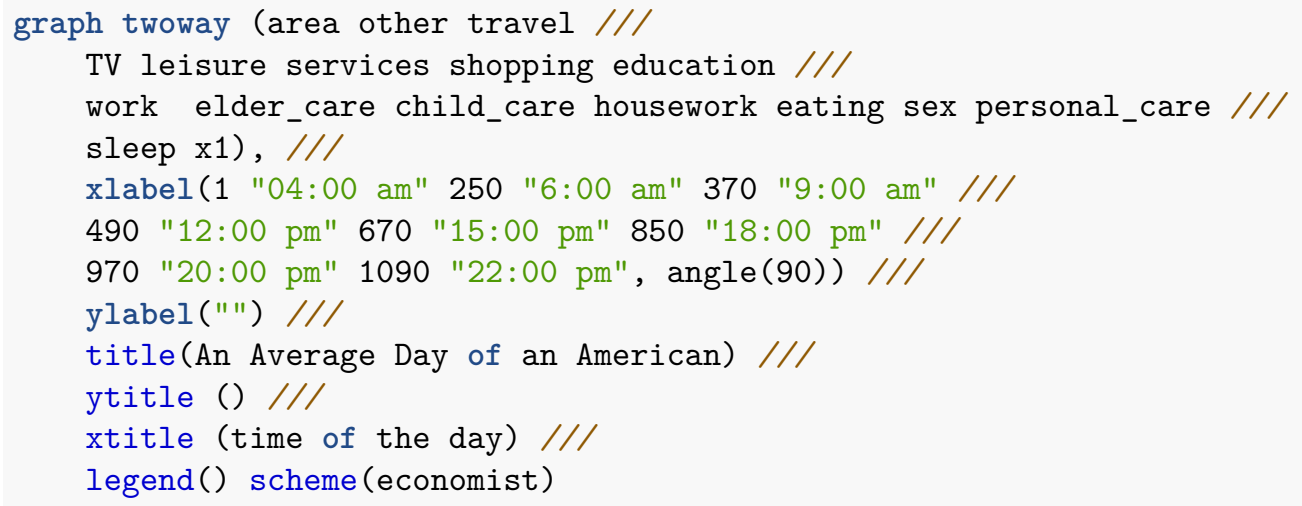

In this example, I used the economist style scheme (see Figure 3).

\section{Example 2. Plotting a Select Activity}

- to plot only one activity, it's better to use non-aggregated activity variables (a1-a15)

For example, to plot sleeping patterns (Figure 4), use:

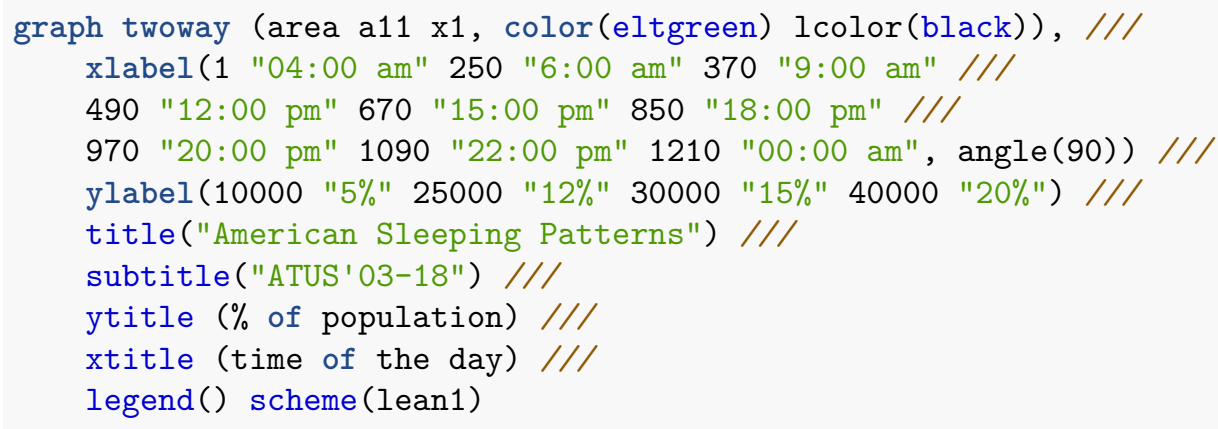

\section{Example 3. Plotting Two Activities}

- to compare two activities, also use non-aggregated activity variables (see Figure 5)

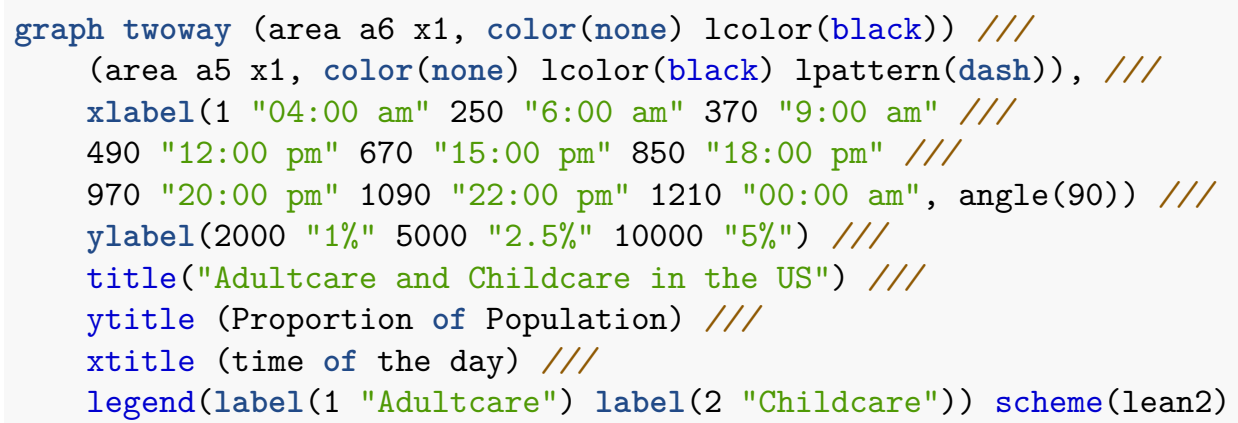

\section{Example 4. Plotting Tempograms for Weekday Sub-sample by Year}

The following example makes use of the variables from atussum0318, particularly weekday and year information. 


\section{An Average Day of an American}
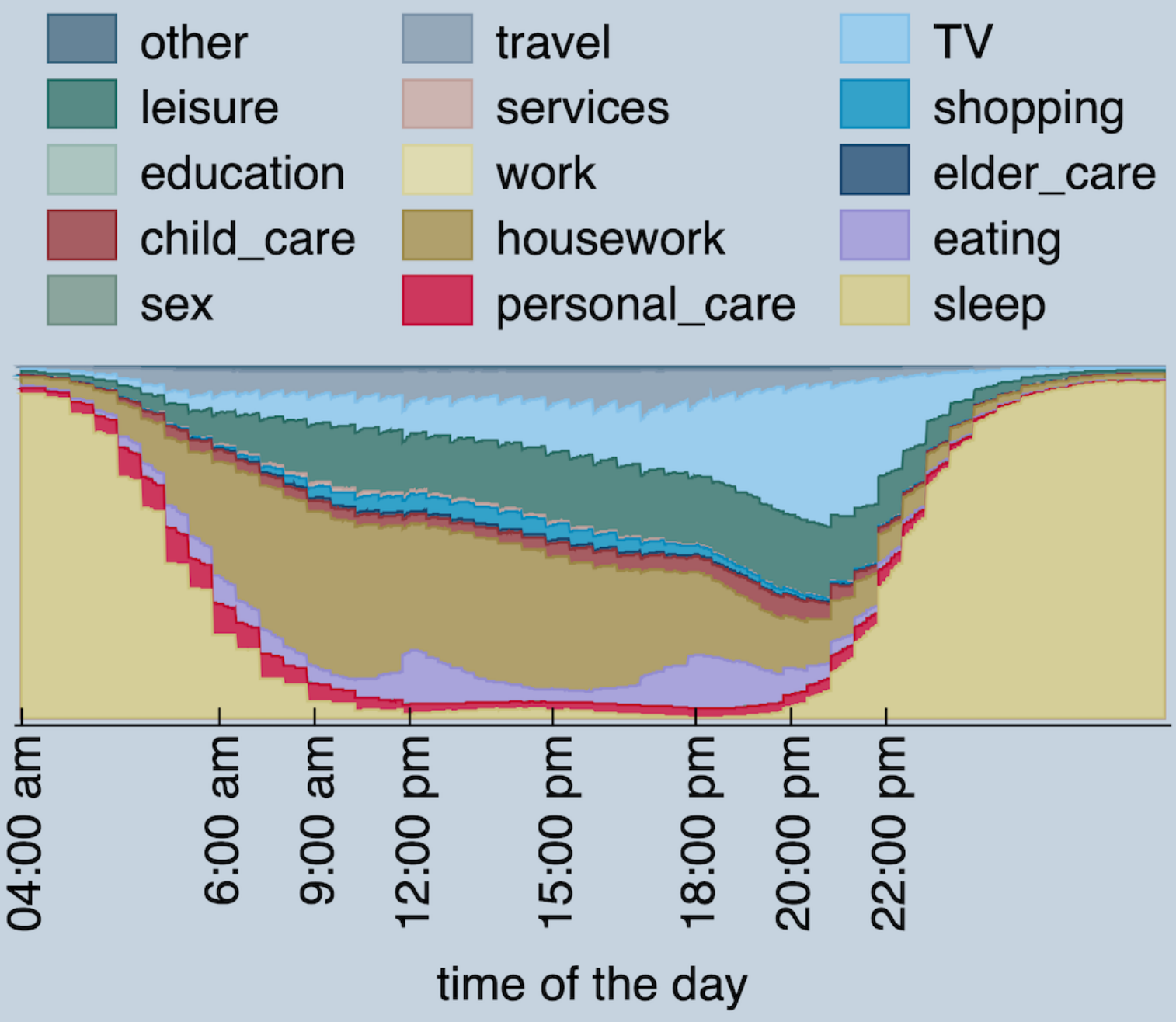

Figure 3: Tempograms: All 


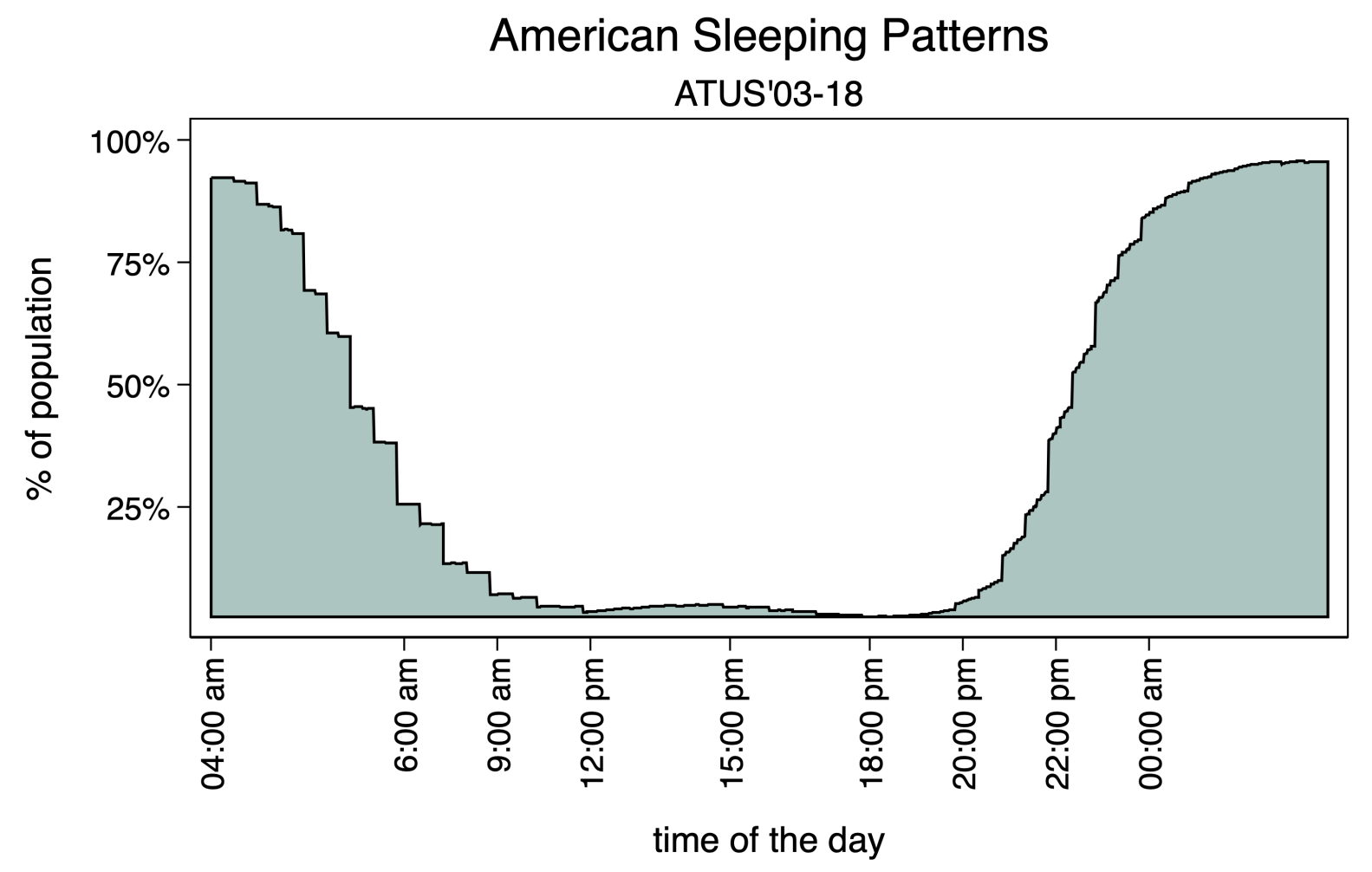

Figure 4: Tempograms: All 


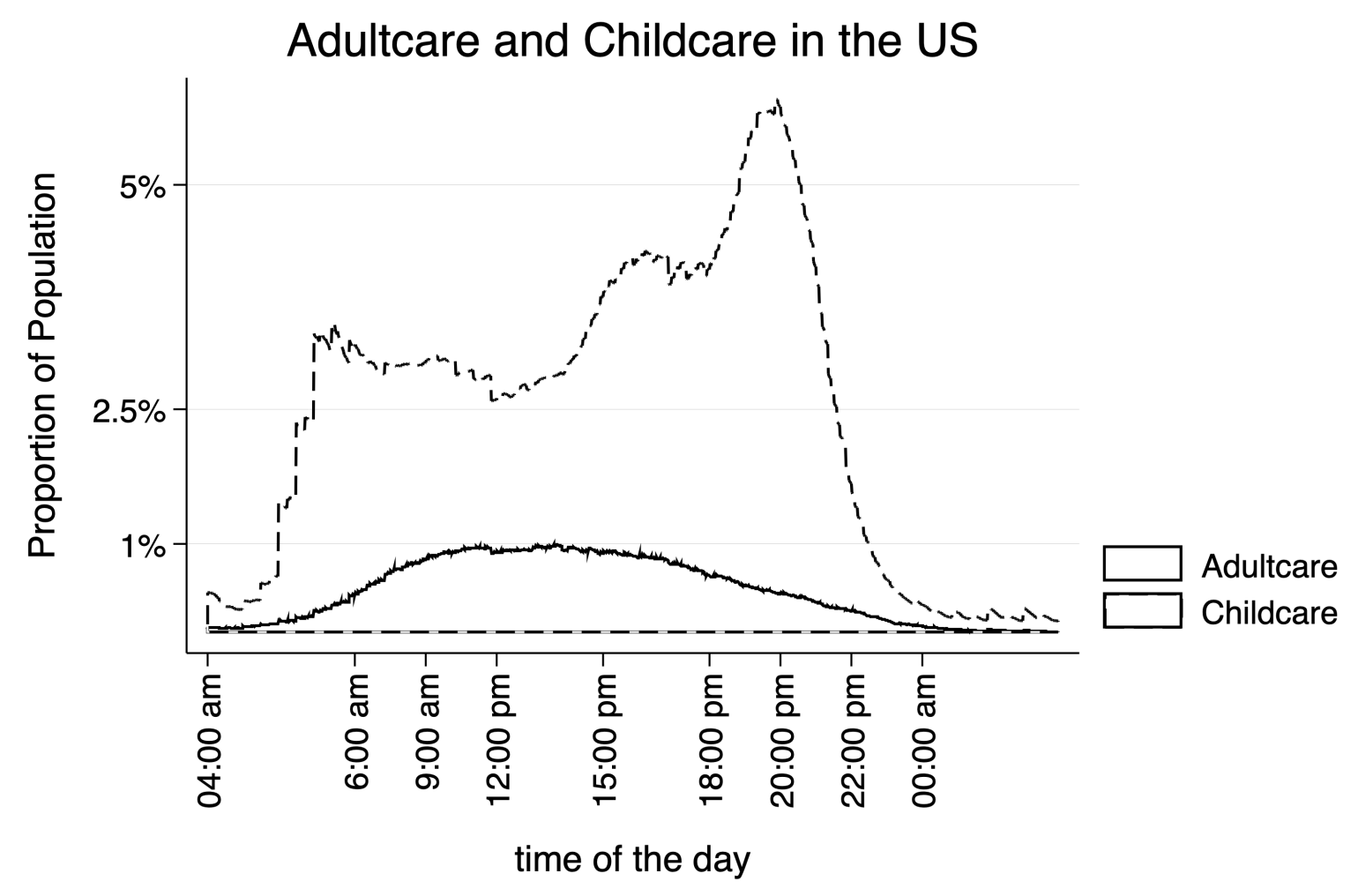

Figure 5: Tempograms: All 
- in order to proceed, first, we need to change the code in Step 11

See Figure 6.

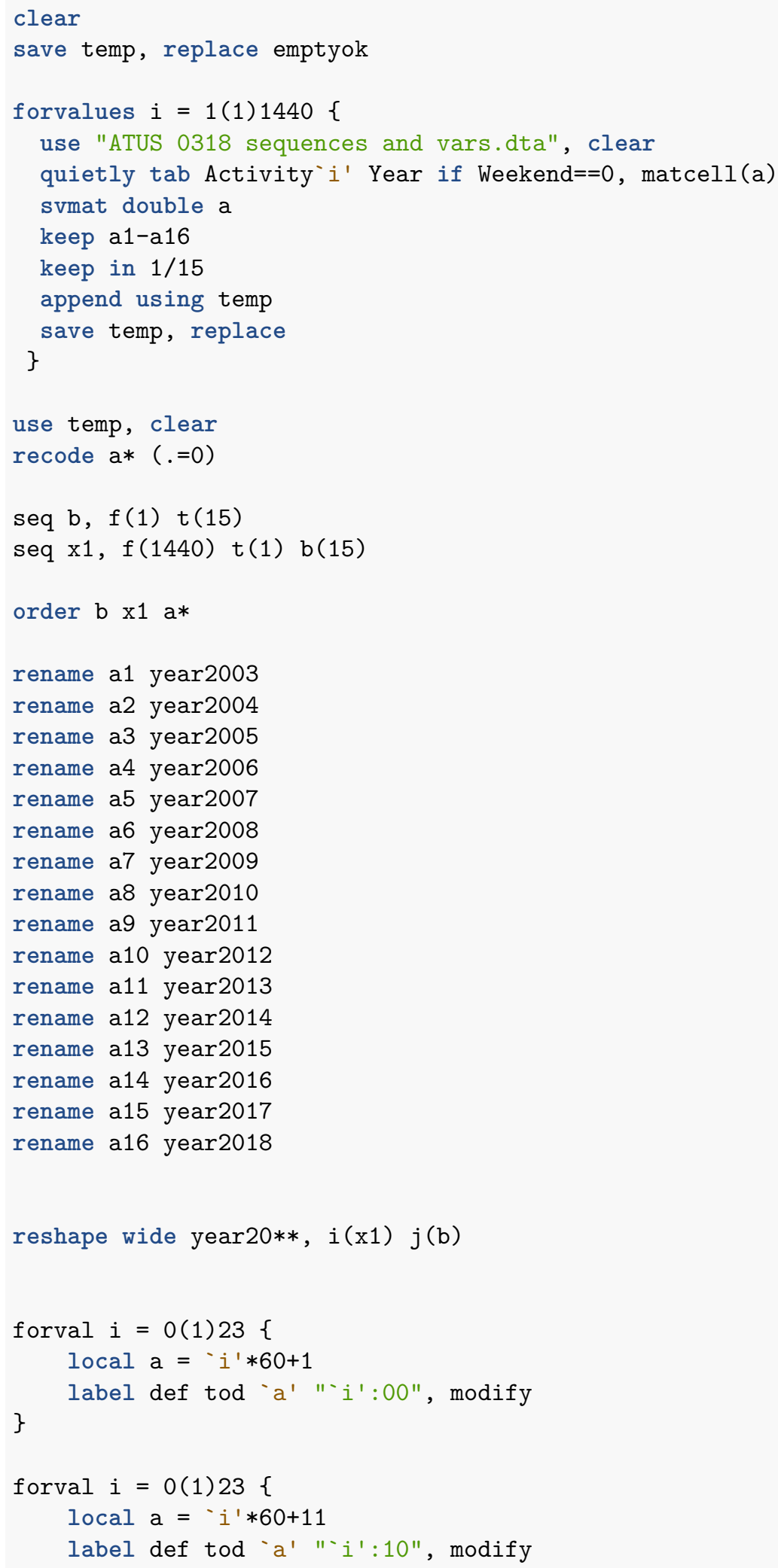




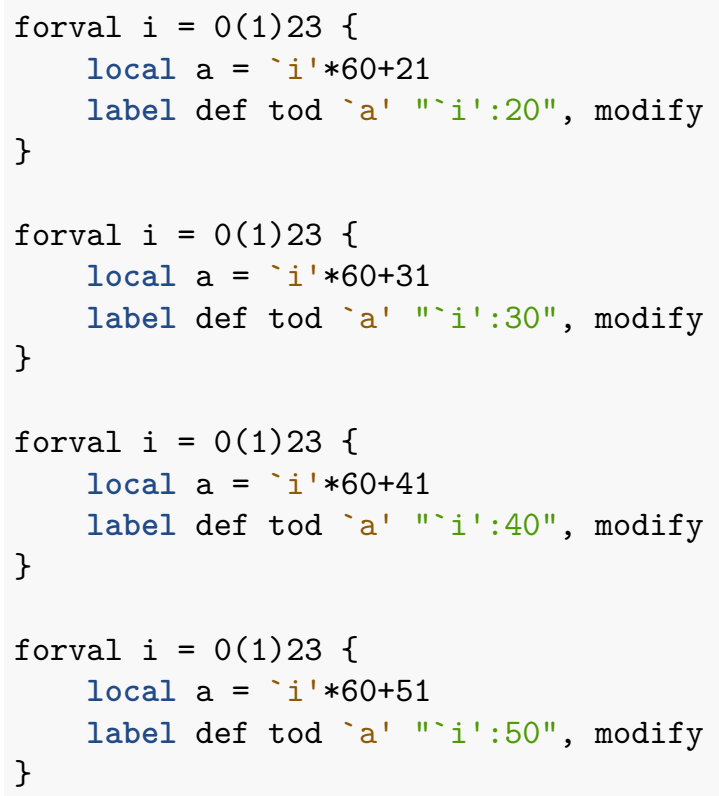



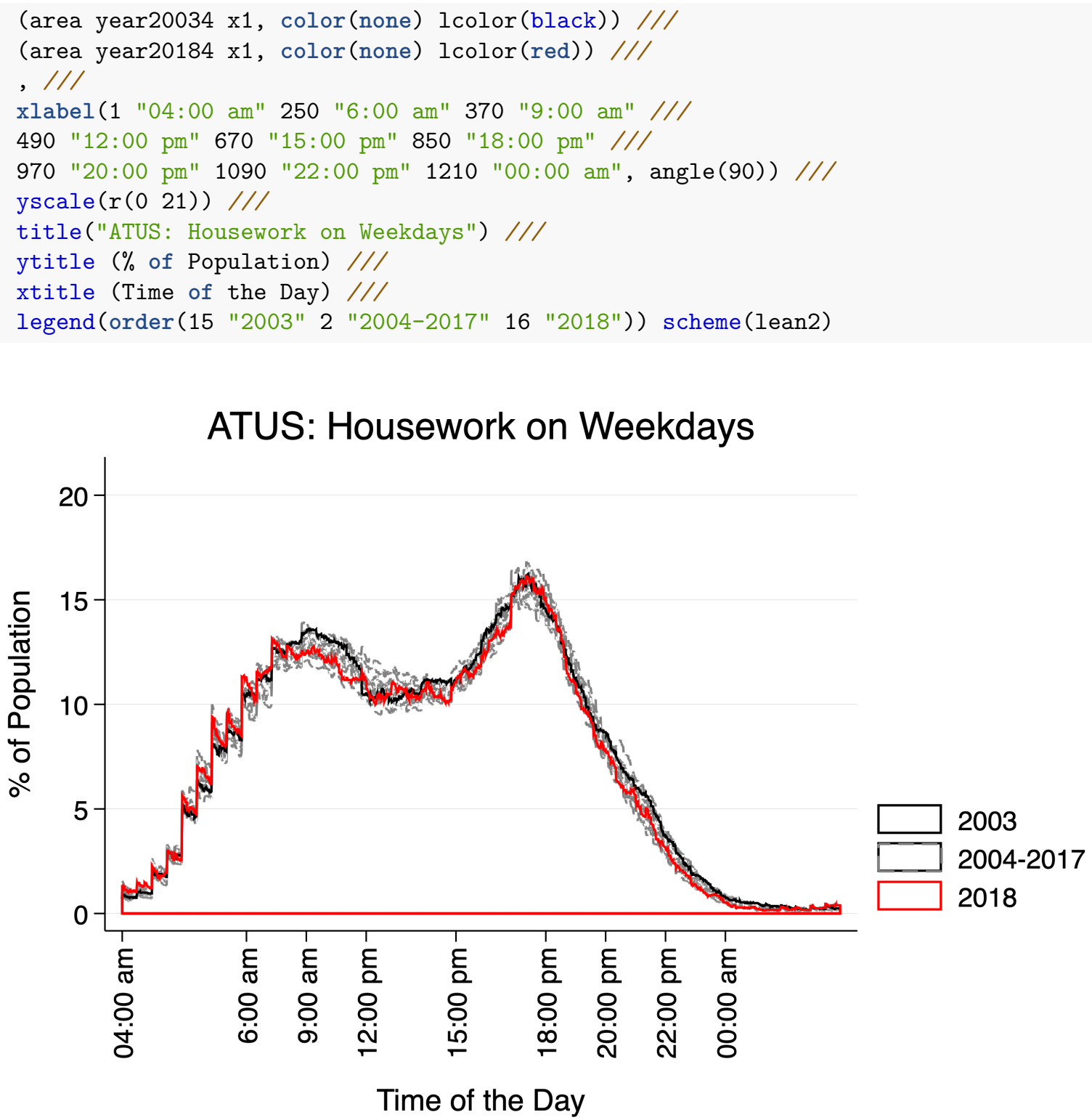

Figure 6: Tempograms: 2003-2018 on Weekdays

.do file can be found on GitHub: https://github.com/Kolpashnikova/How-to-Make-Tempograms-in-Stata If you have questions about the code, please contact the author. 